\title{
Computed tomography in postpartum hemorrhage due to incomplete rupture of an unscarred uterus
}

\author{
Shunya Sugai ${ }^{1}$ (i) $\cdot$ Ikunosuke Tsuneki $^{1} \cdot$ Toru Yanase $^{1}$ (]) $\cdot$ Takumi Kurabayashi $^{1}$ (i)
}

Received: 15 January 2022 / Accepted: 11 February 2022 / Published online: 18 February 2022

(c) The Author(s) 2022

Keywords Computed tomography $\cdot$ Postpartum hemorrhage $\cdot$ Uterine rupture

\section{Description}

Patient 1 was a 33-year-old woman, gravida 2 para 1 , who vaginal delivery at 40 weeks' gestation. Patient 2 was a 37 -year-old woman, gravida 2 para 1 , who vaginal delivery at 39 weeks' gestation. Neither case had undergone uterine surgery, including cesarean section. Both patients developed severe postpartum hemorrhage, but not hemorrhagic shock. They did not complain of abdominal pain. Ultrasonography showed heterogeneous echogenicity in the uterus and no hemoperitoneum. Computed tomography showed extravasation from the myometrium and hematoma formation in the broad ligament (Figs. 1a, b and 2a, b). We performed exploratory laparotomy and identified incomplete uterine rupture in both cases; both recovered well with suture repair (Figs. 1c, d and 2c, d).
Uterine rupture is a rare and life-threatening event. Uterine rupture can be divided into complete rupture, which involves all layers of the uterine wall, and incomplete rupture, in which the visceral peritoneum or broad ligament is left intact. Generally, uterine rupture occurs in patients with a scarred uterus, including those with a history of cesarean section; one study showed that uterine rupture occurred in $0.46 \%$ of patients with a scarred uterus [1]. Rupture of an unscarred uterus can also occur, but this is even rarer. The reported risk factors for rupture of an unscarred uterus include induction of labor with oxytocin or prostaglandins, breech extraction, age of $>35$ years, parity of $>3$, and birthweight of $>4000 \mathrm{~g}$ [2]. Incomplete rupture of an unscarred uterus is difficult to diagnose, but it can be identified by computed tomography.

Shunya Sugai

sugoi3215@gmail.com

1 Department of Obstetrics and Gynaecology, Niigata

City General Hospital, 463-7, Shumoku, Chuo-ku,

Niigata 950-1197, Japan 
Fig. 1 Patient 1: a unenhanced and $\mathbf{b}$ contrast-enhanced computed tomography images obtained during the arterial phase demonstrating extravasation (large arrow). The contrast medium used during uterine artery embolization is visible (small arrows). A hematoma (asterisk) and free air (arrowhead) can be seen in the broad ligament. An intrauterine balloon tamponade device was placed. c Hematomas spreading in the broad ligaments (blue arrowheads). d The broad ligament was incised, and a $10 \mathrm{~cm}$ laceration on the right side of the lower uterus was sutured (blue arrows)
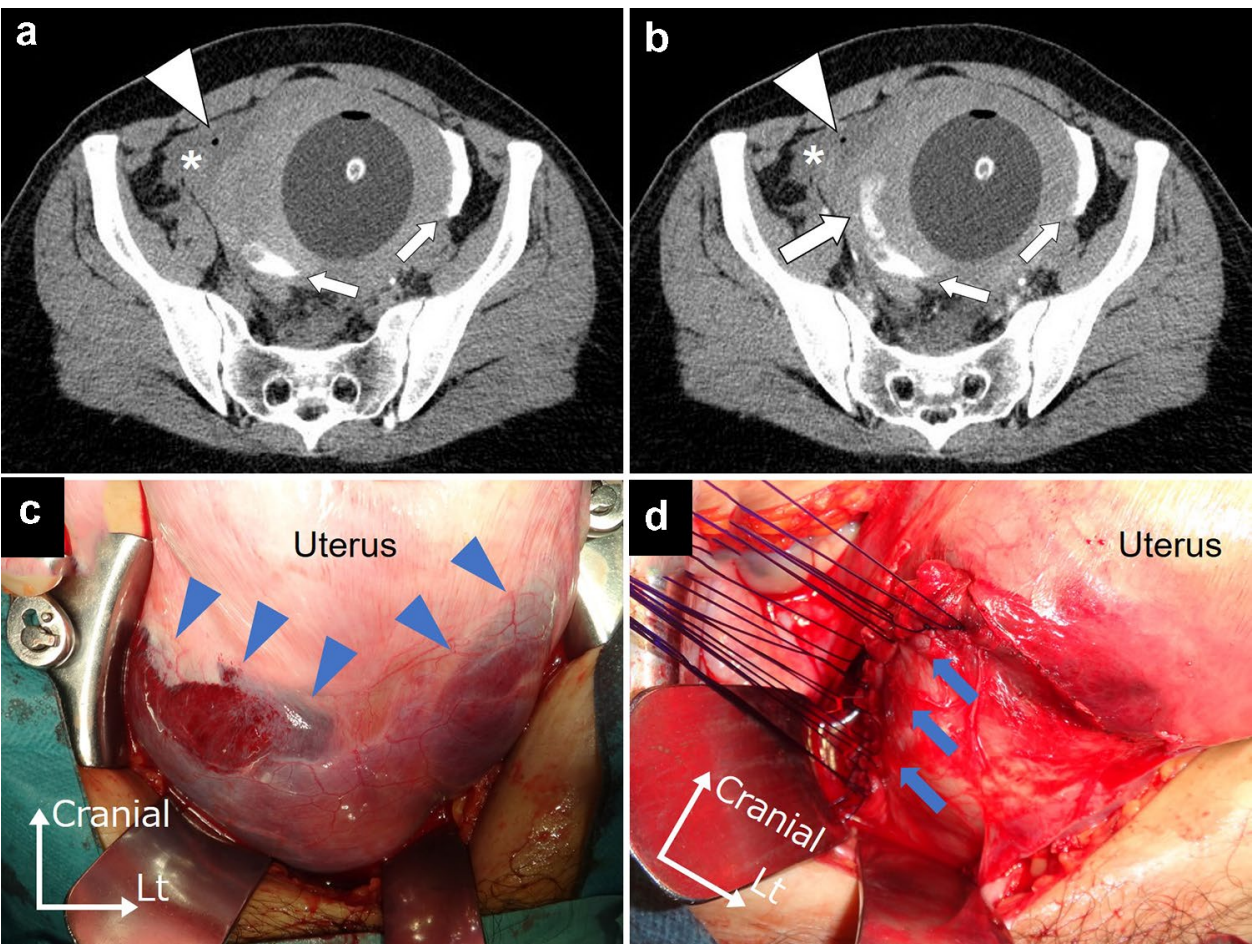

Fig. 2 Patient 2: a, b contrastenhanced computed tomography images obtained during the arterial phase demonstrating extravasation (large arrow). The small arrow indicates iodoform gauze. A hematoma (asterisks) can be seen in the broad ligament. An intrauterine balloon tamponade device was placed. c Hematoma spreading in the broad ligament (blue arrowheads). $\mathbf{d}$ The broad ligament was incised, and an $8 \mathrm{~cm}$ laceration on the left lower uterus was sutured (blue arrows)
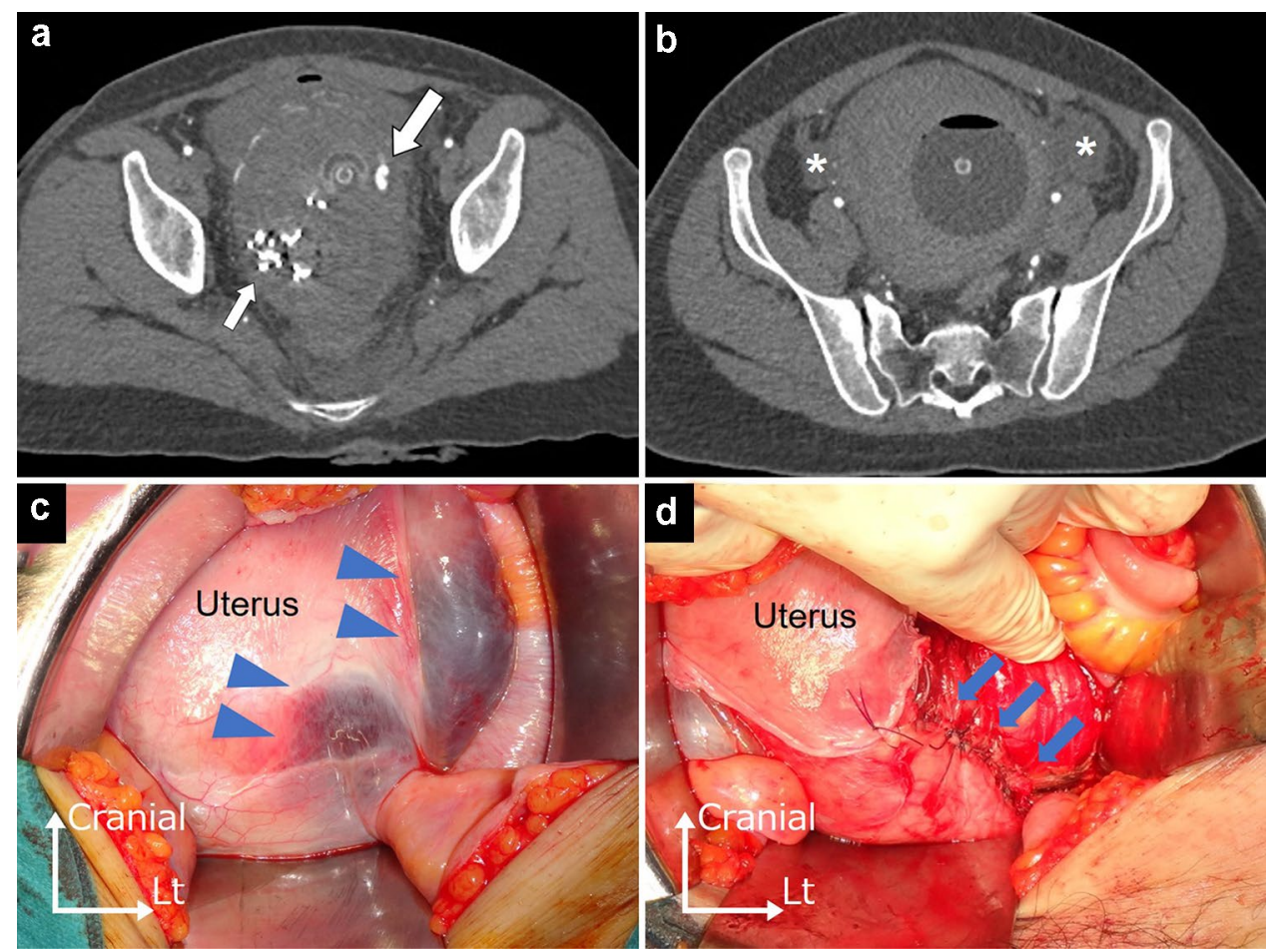

Author contributions SS: data collection, data analysis, manuscript writing, manuscript editing. IT: data collection, manuscript editing. TY: data collection, manuscript editing. TK: data collection, manuscript editing.
Funding Not applicable.

Availability of data and materials All data related to this report are available from the corresponding author on reasonable request. 


\section{Declarations}

Conflict of interest The authors declare that they have no competing interests.

Ethics approval Our facility determined that ethics approval was not required.

Consent to participate Informed consent was obtained from the patient.

Consent for publication Informed consent for publication was obtained from the patient.

Open Access This article is licensed under a Creative Commons Attribution 4.0 International License, which permits use, sharing, adaptation, distribution and reproduction in any medium or format, as long as you give appropriate credit to the original author(s) and the source, provide a link to the Creative Commons licence, and indicate if changes were made. The images or other third party material in this article are included in the article's Creative Commons licence, unless indicated otherwise in a credit line to the material. If material is not included in the article's Creative Commons licence and your intended use is not permitted by statutory regulation or exceeds the permitted use, you will need to obtain permission directly from the copyright holder. To view a copy of this licence, visit http://creativecommons.org/licenses/by/4.0/.

\section{References}

1. Takeya A, Adachi E, Takahashi Y, Kondoh E, Mandai M, Nakayama T (2020) Trial of labor after cesarean delivery (TOLAC) in Japan: rates and complications. Arch Gynecol Obstet 301:995-1001

2. Al-Zirqi I, Daltveit AK, Forsén L, Stray-Pedersen B, Vangen S (2017) Risk factors for complete uterine rupture. Am J Obstet Gynecol 216:165.e1-165.e8

Publisher's Note Springer Nature remains neutral with regard to jurisdictional claims in published maps and institutional affiliations. 